\title{
Receiver operating characteristic analysis to determine optimal fluid management during open colorectal surgery
}

\author{
B. Pache* (D, M. Hübner*, J. Solà†, D. Hahnloser*, N. Demartines* and F. Grass* \\ *Department of Visceral Surgery, Lausanne University Hospital CHUV, Lausanne, Switzerland, and †Centre Suisse d'Electronique et de Microtechnique \\ (CSEM), Neuchâtel, Switzerland
}

Received 7 July 20 I8; accepted 3 October 20 18; Accepted Article Online 8 November 2018

\begin{abstract}
Aim The present study aimed to analyse fluid management and to define optimal fluid-related thresholds for elective open colorectal surgery.

Method A retrospective analysis was made of all consecutive elective open colorectal resections performed in our tertiary centre between May 2011 and May 2017. The main outcomes were postoperative complications [overall (I-V) and severe (IIIB-V) according to the Clavien classification], respiratory complications and postoperative ileus (POI). Critical thresholds regarding perioperative fluid management and postoperative weight gain were identified by using receiver operator characteristic (ROC) analysis. Independent risk factors for overall complications were identified by multivariable logistic regression analysis.
\end{abstract}

Results Of 121 patients who had open operations, 84 $(69 \%)$ had some complication and $26(21 \%)$ had severe complications. Respiratory complications and POI occurred in $15(12 \%)$ and 46 patients (38\%), respectively. The thresholds for intravenous fluids were 3.51 at postoperative day (POD) 0 [area under ROC curve (AUROC) 0.7 for any 0.69 for respiratory complications] and $3.5 \mathrm{~kg}$ weight gain at POD 2 (AUROC
0.82 for respiratory complications). Multivariable analysis revealed weight gain of $>3.5 \mathrm{~kg}$ at POD 2 (OR $5.9 ; 95 \%$ CI 1.3-16.6) as a significant risk factor for overall complications. Acute kidney injury was observed in five patients $(4 \%)$, three $(5 \%)$ in the group with $>3.51$ at POD 0 and two $(3 \%)$ in the group with $<3.51$ at $\mathrm{POD} 0(P=0.64)$. Creatinine increase was transitory and all patients regained baseline levels before discharge.

Conclusion A weight gain of $>3.5 \mathrm{~kg}$ at POD 2 has been identified as the critical threshold for overall and respiratory complications and prolonged length of stay after open elective colorectal surgery.

Keywords Open colectomy, fluid management, weight gain, postoperative morbidity, postoperative complications, ileus

\section{What does this paper add to the literature?}

Stringent intra-operative fluid management during elective open colorectal surgery is critical in order to reduce postoperative adverse events. This study identified weight gain of $3.5 \mathrm{~kg}$ at postoperative day 2 as the critical threshold to help with guidance in clinical practice.

\section{Introduction}

In many countries the open approach for colorectal resection has been steadily replaced by minimally invasive techniques with improved patient recovery and reduced length of stay as well as decreased postoperative short-term morbidity $[1,2]$. Nevertheless, the open approach remains inevitable in some circumstances such as an unfavourable patient or pathological or surgery-

Correspondence to: Professor N. Demartines, Department of Visceral Surgery, Lausanne University Hospital CHUV, Bugnon 46, I0II Lausanne, Switzerland. E-mail: demartines@chuv.ch related conditions [3]. Enhanced recovery after surgery (ERAS) protocols advocate adherence to near zero fluid balance, since excessive fluid administration has repeatedly been linked to adverse postoperative outcomes [4-6].

Specific thresholds regarding fluid management have not yet been investigated, particularly after open colorectal surgery, which still represents the predominant approach worldwide [7].

The aim of the present study was to identify thresholds with regard to perioperative intravenous (IV) fluid replacement and postoperative weight gain after open elective colorectal resections. 


\section{Method}

\section{Patients}

All consecutive elective open colorectal resections at Lausanne University Hospital (CHUV) between May 2011 and May 2017 were included. Patients were treated according to a comprehensive ERAS pathway [8]. Prospective collection of data was ensured by a dedicated ERAS-trained clinical nurse. Data accuracy was reviewed during regular audit sessions by the institutional ERAS team. Contents of the ERAS database have previously been described [9].

All types of open colonic and rectal resections, including low anterior resection, proctocolectomy and abdominoperineal excision were included. Laparoscopic procedures were excluded, as were laparoscopic conversions on the basis of an intention-to-treat principle. Also excluded were stoma formation and Hartmann's reversal.

\section{Ethical considerations}

All data were retrieved from the ERAS International Audit System (EIAS), which is our prospective online database. Data were anonymized prior to analysis. This study was approved by the institutional review board (Commission cantonale d'éthique de la recherche sur l'être humain CER-VD no. 201701971) and conducted according to the STROCSS criteria [10].

\section{Assessment of perioperative fluid management and postoperative weight}

Thresholds were identified for three fluid-management related parameters through receiver operator characteristic (ROC) analysis, with the pragmatic considerations of it being easy to remember and of clinical usefulness (results are to be obtained as easily as possible to launch subsequent treatments):

(1) total IV volume administered on the day of surgery [= postoperative day $(\mathrm{POD}) 0]$, including intraoperatively administered liquids such as crystalloids, colloids, blood products and postoperative IV liquids

(2) postoperative weight was assessed on PODs 1-3 using standard scales for every patient

(3) the amount of weight- and duration-adjusted intraoperative IV fluids, calculated in $\mathrm{ml} / \mathrm{kg} / \mathrm{h}$, with balanced administration being defined as $<7 \mathrm{ml} /$ $\mathrm{kg} / \mathrm{h}[11]$.

\section{Outcomes/study end-points}

Complications were classified according to the Clavien classification score [12]. The primary end-point was the overall complication rate (Clavien grade I-V). Secondary end-points were major complications (Clavien IIIb-V), respiratory complications, postoperative ileus and length of stay. Respiratory complications were defined as [13]:

(1) radiologically confirmed pneumonia (needing antibiotic treatment)

(2) lobar atelectasis (needing physiotherapeutic treatment beyond standard use of incentive spirometry six times a day)

(3) pleural effusions (needing surgical or radiologically guided thoracic drainage)

(4) respiratory failure needing transfer to an intermediate or intensive care unit (ICU).

Postoperative ileus was defined as time to first stool beyond POD 3 [14]. Acute kidney injury was defined according to 'risk, injury, failure, loss of kidney function and end-stage kidney disease' (RIFLE) [15]. Of note, nonsteroidal anti-inflammatory drugs were not part of standardized postoperative pain management within the enhanced recovery protocol. Instead, metamizole was used.

All end-points were assessed in hospital and until POD 30 (outpatient control visit). All outcomes were compared between patients above and below the thresholds specified above.

Length of hospital stay and overall compliance to the ERAS protocol of $>70 \%$ were assessed $[16,17]$.

\section{Statistical analysis}

Thresholds were identified through ROC analysis to jointly yield the optimal sensitivity and specificity of each ROC curve. The ROC curves were calculated with the Statistical and Machine Learning Toolbox of MATLAB R2018a (Mathworks, Natick, Massachusetts). Descriptive statistics for categorical variables were reported as frequency (\%), while continuous variables were reported as mean (standard deviation) or median (interquartile range). Chi-square was used for comparison of categorical variables. All statistical tests were two-sided, and a level of 0.05 was used to indicate statistical significance. Data analysis was performed with the Statistical Software for the Social Sciences SPSS Advanced Statistics 22 (IBM Software Group, Chicago, Illinois, USA).

\section{Results}

\section{Patients}

The study cohort included 121 consecutive patients. Overall complications (Clavien I-V) were observed 
in 84 patients $(69 \%)$. Demographic and surgical data comparing patients with and without overall complications are shown in Table 1. Twenty-six patients $(21 \%)$ had severe complications (Clavien IIIb-V), 15 patients (12\%) had respiratory complications and 46 patients (38\%) had postoperative ileus. Acute kidney injury was observed in five patients $(4 \%)$, three $(5 \%)$ in the group receiving $>3.51$ at POD 0 and two $(3 \%)$ in the group with $<3.51$ at POD $0 \quad(P=0.64)$. Creatinine increase was transitory, and all patients regained baseline levels before discharge.

\section{Thresholds for fluid administration and postoperative weight gain}

The ROC curves that were used to set thresholds for total IV fluids at POD 0 and postoperative weight gain are available in the online Supporting Information (Appendix S1).

Thresholds according to ROC curves were set as follows: 3.51 of IV fluids at POD 0 [area under the ROC curve (AUROC) 0.7 for any/0.69 for respiratory complications, negative predictive value (NPV) $70 \%$ and $94 \%$, respectively) and weight gain of $3.5 \mathrm{~kg}$ at POD 2 (AUROC 0.82 for respiratory complications, NPV $98 \%)$.

\section{Comparison of outcomes in patients below and above the threshold}

Figure 1 gives an overview on all outcomes including length of stay for patients below and above thresholds (IV fluids and weight).

\section{Independent factors associated with overall complications}

Multivariable analysis retained ERAS compliance of $>70 \%$ as a protective factor (OR $0.2 ; 95 \%$ CI $0.1-0.8$ ) and weight gain of $>3.5 \mathrm{~kg}$ at POD 2 (OR 5.9; 95\% CI 1.3-16.6) as risk factors for overall complications, while fluid administration of $>3.51$ at POD 0 was not found to be an independent risk factor for overall complications (Fig. 2).

\section{Discussion}

Fluid administration of 3.51 at POD 0 and weight gain of $3.5 \mathrm{~kg}$ at POD 2 were identified through ROC analysis as critical thresholds for complications in elective open colorectal surgery. Weight gain of $>3.5 \mathrm{~kg}$ was independently associated with overall complications, and patients exceeding either threshold had significantly more respiratory complications and prolonged length of

Table I Demographics.

\begin{tabular}{|c|c|c|c|c|}
\hline & $\begin{array}{l}\text { All patients } \\
(n=121)\end{array}$ & $\begin{array}{l}\text { No complication } \\
(n=37)\end{array}$ & $\begin{array}{l}\text { Any complication } \\
(n=84)\end{array}$ & $P$ \\
\hline Age $($ mean $\pm \mathrm{SD})$ & $63 \pm 16$ & $62 \pm 15$ & $64 \pm 16$ & 0.62 \\
\hline Age $\geq 70$ years $(\%)$ & $44(36)$ & $11(30)$ & $33(39)$ & 0.41 \\
\hline Gender $(M: F)$ & $56: 65$ & $18: 19$ & $38: 46$ & 0.84 \\
\hline $\operatorname{BMI}\left(\mathrm{kg} / \mathrm{m}^{2}\right)($ mean $\pm \mathrm{SD})$ & $25.3 \pm 5.7$ & $25.9 \pm 5$ & $25.1 \pm 6$ & 0.48 \\
\hline ASA group $(1-2: 3-4)(\%: \%)$ & $78: 43(64: 36)$ & $29: 8(78: 22)$ & $49: 35(58: 42)$ & 0.04 \\
\hline Previous abdominal surgery (\%) & $86(71)$ & $26(70)$ & $60(71)$ & 1 \\
\hline Malignancy (\%) & $94(78)$ & $25(68)$ & $69(82)$ & 0.08 \\
\hline Rectal procedure (\%) & $46(38)$ & $11(30)$ & $35(42)$ & 0.23 \\
\hline Operation duration $(\min )($ mean $\pm S D)$ & $250 \pm 120$ & $200 \pm 130$ & $270 \pm 120$ & 0.007 \\
\hline Operation duration $>180 \mathrm{~min}(\%)$ & $76(63)$ & $15(41)$ & $61(73)$ & 0.001 \\
\hline \multicolumn{5}{|l|}{ IV fluid administration } \\
\hline Total at POD $0(\mathrm{ml})($ mean $\pm \mathrm{SD})$ & $3900 \pm 2100$ & $3000 \pm 1300$ & $4300 \pm 2300$ & $<0.001$ \\
\hline Total at POD $0>3.51(\%)$ & $59(49)$ & $11(30)$ & $48(57)$ & 0.006 \\
\hline Intra-operative fluid administration $>7 \mathrm{ml} / \mathrm{kg} / \mathrm{h}(\%)$ & $93(78)$ & $26(72)$ & $67(80)$ & 0.47 \\
\hline ERAS compliance $>70 \%(\%)$ & $56 / 96(58)$ & $23 / 28(82)$ & $33 / 68(49)$ & 0.003 \\
\hline Weight gain at POD $2(\mathrm{~kg})($ mean $\pm \mathrm{SD})$ & $2.7 \pm 2.6$ & $2 \pm 2.1$ & $3.1 \pm 2.8$ & 0.06 \\
\hline Weight gain $>3.5 \mathrm{~kg}$ at POD $2(\%)$ & $28 / 81(35)$ & $4 / 29(14)$ & $24 / 52(46)$ & 0.004 \\
\hline
\end{tabular}

Baseline demographic and surgical parameters of patients without complications $(n=37)$ and patients with any complication (Clavien I-V) $(n=84)$.

Bold characters indicate significant values $(P<0.05)$.

BMI, body mass index. 
(a) IV fluids

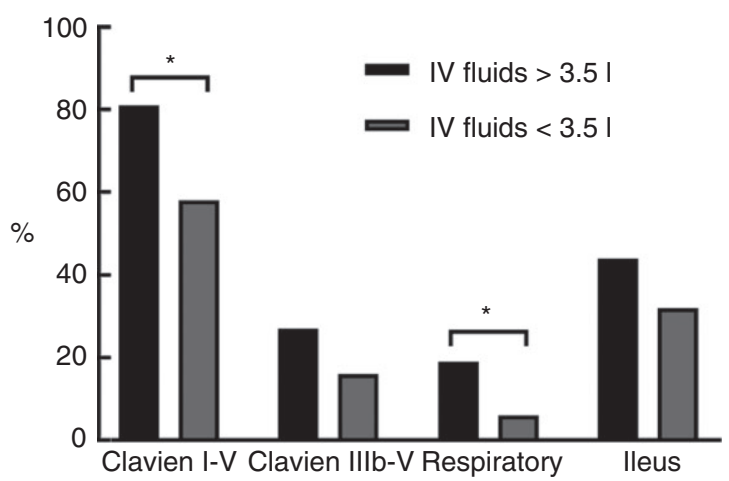

(b) Weight

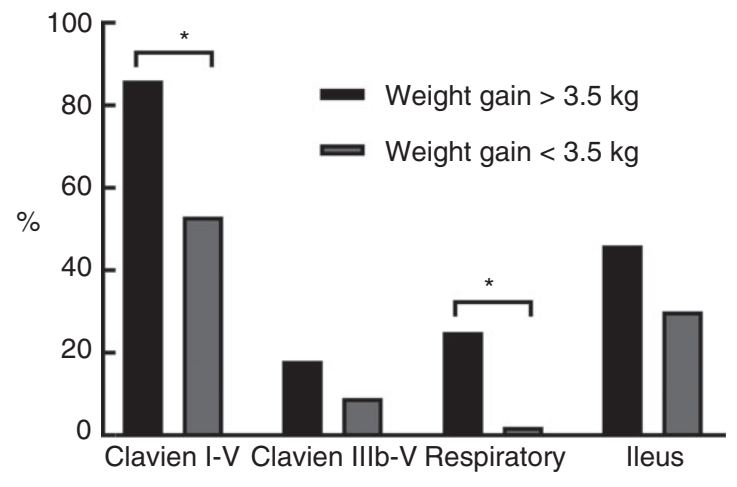

(c) Length of stay

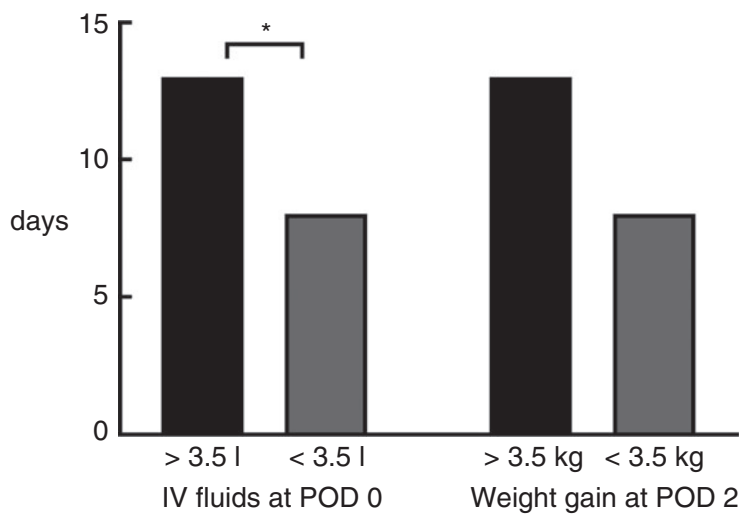

Figure I Thresholds regarding outcome: (a) IV fluids, (b) weight and (c) length of stay. Comparison of percentages of overall complications (Clavien I-V, $n=84$ ), major complications (Clavien IIIb-V, $n=26$ ), respiratory complications $(n=15)$ and postoperative ileus $(n=46)$ in (a) patients receiving $>3.51$ of total fluids at POD 0 and patients receiving $<3.51$ and (b) patients gaining $>3.5 \mathrm{~kg}$ of body weight at POD 2 and patients gaining $<3.5 \mathrm{~kg}$. Comparison of length of hospital stay (c) in patients receiving $>3.51$ of total fluids at $\mathrm{POD} 0 />3.5 \mathrm{~kg}$ of body weight at POD 2 (black bars) and patients receiving $<3.5 \mathrm{l} /$ gaining $<3.5 \mathrm{~kg}$ (grey bars). *Indicates statistical significance $(P<0.05)$. hospital stay. POD 0 fluids of 3.51 , however, was not retained as an independent risk factor for overall complications.

Even though stringent fluid management to prevent postoperative complications is a widely accepted concept in patients undergoing elective surgery, no thresholds are available for guidance $[6,18]$. A wide variability of intra-operative fluid administration was described in a single-centre analysis of a colorectal surgical case-mix before implementation of goal-directed fluid therapy [19]. A recent cohort study analysed 911 consecutive colorectal cancer patients in a Swedish ERAS cohort by defining a restrictive fluid regimen as $<31$ at POD 0 [20], but ROC analyses were not performed. Patients below this threshold had fewer short-term complications, shorter length of stay and even improved 5-year survival. A large observational study reporting average fluid administration of 3.21 at POD 0 found similarly a strong positive association between POD 0 fluids and length of hospital stay in patients with varying surgical risk profiles undergoing colorectal surgery [21].

Fluid overload was associated with adverse events postoperatively in many large-scale studies $[22,23]$. On the other hand, a recent study showed that restrictive fluid management resulted in a higher rate of acute kidney injury [24]. In fact, half of the patients were not treated within enhanced recovery protocols. The subgroup treated within the ERAS protocol displayed no kidney injury, thus emphasizing the importance of a global patient management strategy with restrictive fluid management but within an ERAS pathway.

The present study focused on IV fluid administration rather than urine output as a guidance parameter, since low urine output by itself is no longer considered harmful $[25,26]$. Srinivasa et al. described no difference in urine output between patients monitored by goal-directed fluid therapy (GDFT) and patients with restrictive fluid management [27]. Interestingly, the same group found that GDFT through invasive monitoring was not easily accessible due to lack of availability or experience with monitoring tools [28]. A recent randomized trial by Puckett et al. found that a threshold of $0.2 \mathrm{ml} / \mathrm{kg} /$ $\mathrm{h}$ with IV fluid sparing was not inferior to the standard target of $0.5 \mathrm{ml} / \mathrm{kg} / \mathrm{h}$ [29].

As an additional measure, laparoscopic surgery provides several advantages that result in faster recovery [30]. Surgical trauma is less pronounced, and fluid shifts through the open abdomen and blood loss are lower compared with open surgery [31]. A minimally invasive approach has been widely adopted as the standard approach for colorectal resections, but fluid management in open surgery presents different challenges. 


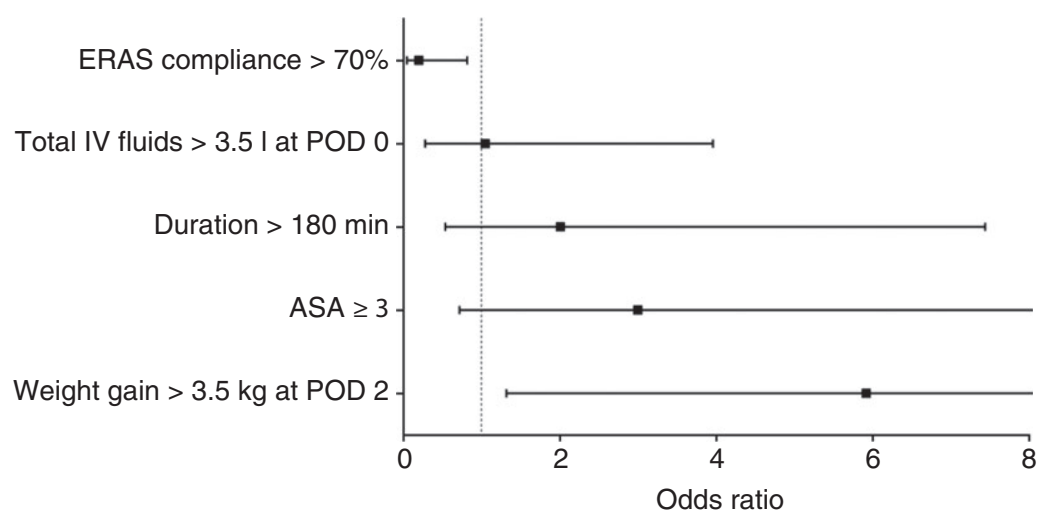

Figure 2 Multivariable analysis of univariate factors with $P<0.05$ associated with any complication (Clavien I-V). An odds ratio of more than 1 increases the risk of any complication.

The thresholds identified in the present study might serve as guidelines specifically for open colorectal surgery. Fluid administration for open cases might be difficult to standardize, and the univariate association between POD 0 fluids of 3.51 and complications vanished in the multivariable model. This model included operation duration and American Society of Anesthesiologists score, both surrogate parameters for surgical and anaesthetic difficulty. On the other hand, POD 2 weight gain of $3.5 \mathrm{~kg}$ was independently associated with overall complications and might even be a better indicator of fluid excess after the initial recovery period of 24-48 h. Ambulation, fluid restriction and preemptive diuretic treatment may be launched or intensified at this stage. The independent beneficial effect of overall compliance to the ERAS protocol may also emphasize this conclusion. With this in mind, fluid management needs to be embedded in the assessment of overall compliance $[16,17]$.

Patients above critical thresholds presented a significant increase in respiratory complications, which are of particular concern after open surgery [32]. The rate of $12 \%$ in the present cohort, however, is within the reported range [33]. Fluid administration below the threshold had a particularly strong positive impact on respiratory complications in the present study, while further complications did not differ significantly between the two groups, which was likely to be due to aType II error (small sample size; Fig. 1).

Several limitations of the present study need to be discussed. Even though data were obtained prospectively in a fully implemented ERAS pathway, analysis was performed retrospectively, with its inherent limitations. As laparoscopy is the standard approach for colorectal surgery in our institution $79 \%$ of elective colorectal surgery was performed by laparoscopy during the study period) rather few patients were available for analysis. As a consequence, only patients who were not suitable for a laparoscopic approach were included, which introduces a selection bias. The reasons for an open approach were mainly surgical history and adhesions impeding abdominal access, as witnessed by the high number of patients who had previous surgery $(>70 \%)$. However, the precise reasons for open surgery were not available. The suggested thresholds are not intended to replace an individual patient-centred approach according to disease presentation, patient characteristics and surgical difficulty, but rather serve as warnings and points of reference.

Independent confirmation of these results is necessary for there to be a wholesale acceptance of the findings.

\section{Conclusions}

Weight at POD 2 might be a predictor for postoperative adverse events after open elective colorectal surgery. A threshold of $3.5 \mathrm{~kg}$ may serve as a reference for clinical guidance to break the vicious cycle of further postoperative fluid overload in a timely manner.

\section{Acknowledgements}

The authors would like to acknowledge the support provided by all members of the enhanced recovery after surgery team in Lausanne.

\section{Conflict of interests}

None declared.

\section{References}

1 Yamada T, Okabayashi K, Hasegawa $\mathrm{H}$ et al. Meta-analysis of the risk of small bowel obstruction following open or 
laparoscopic colorectal surgery. Br J Surg 2016; 103: 493503.

2 Reza MM, Blasco JA, Andradas E, Cantero R, Mayol J. Systematic review of laparoscopic versus open surgery for colorectal cancer. Br J Surg 2006; 93: 921-8.

3 Schlussel AT, Lustik MB, Johnson EK et al. A nationwide assessment comparing nonelective open with minimally invasive complex colorectal procedures. Colorectal Dis 2016; 18: 301-11.

4 Gustafsson UO, Scott MJ, Schwenk W et al. Guidelines for perioperative care in elective colonic surgery: Enhanced Recovery After Surgery $\left(\right.$ ERAS $^{\circledR}$ ) Society recommendations. World J Surg 2013; 37: 259-84.

5 Lobo DN, Bostock KA, Neal KR, Perkins AC, Rowlands BJ, Allison SP. Effect of salt and water balance on recovery of gastrointestinal function after elective colonic resection: a randomised controlled trial. Lancet 2002; 359: 1812-8.

6 Myles PS, Andrews S, Nicholson J, Lobo DN, Mythen M. Contemporary approaches to perioperative IV fluid therapy. World J Surg 2017; 41: 2457-63.

7 Alnasser M, Schneider EB, Gearhart SL et al. National disparities in laparoscopic colorectal procedures for colon cancer. Surg Endosc 2014; 28: 49-57.

8 Roulin D, Donadini A, Gander S et al. Cost-effectiveness of the implementation of an enhanced recovery protocol for colorectal surgery. Br J Surg 2013; 100: 1108-14.

9 Grass F, Slieker J, Frauche P et al. Postoperative urinary retention in colorectal surgery within an enhanced recovery pathway. J Surg Res 2017; 207: 70-6.

10 Agha RA, Borrelli MR, Vella-Baldacchino M, Thavayogan R, Orgill DP, Group S. The STROCSS statement: strengthening the Reporting of Cohort Studies in Surgery. Int J Surg 2017; 46: 198-202.

11 Doherty M, Buggy DJ. Intraoperative fluids: how much is too much? Br J Anaesth 2012; 109: 69-79.

12 Dindo D, Demartines N, Clavien PA. Classification of surgical complications: a new proposal with evaluation in a cohort of 6336 patients and results of a survey. Ann Surg 2004; 240: 205-13.

13 Miskovic A, Lumb AB. Postoperative pulmonary complications. Br J Anaesth 2017; 118: 317-34.

14 Gero D, Gie O, Hubner M, Demartines N, Hahnloser D. Postoperative ileus: in search of an international consensus on definition, diagnosis, and treatment. Langenbecks Arch Surg 2016; 402: 149-58.

15 Wilson T, Quan S, Cheema K et al. Risk prediction models for acute kidney injury following major noncardiac surgery: systematic review. Nephrol Dial Transplant 2016; 31: 23140.

16 Jurt J, Slieker J, Frauche P et al. Enhanced recovery after surgery: can we rely on the key factors or do we need the bel ensemble? World J Surg 2017; 41: 2464-70.

17 Gustafsson UO, Hausel J, Thorell A et al. Adherence to the enhanced recovery after surgery protocol and outcomes after colorectal cancer surgery. Arch Surg 2011; 146: 5717.
18 Group EC. The Impact of Enhanced Recovery Protocol Compliance on Elective Colorectal Cancer Resection: results From an International Registry. Ann Surg 2015; 261: 1153-9.

19 Quinn TD, Brovman EY, Urman RD. Analysis of variability in intraoperative fluid administration for colorectal surgery: an argument for goal-directed fluid therapy. J Laparoendosc Adv Surg Tech A 2017; 27: 892-7.

20 Asklid D, Segelman J, Gedda C, Hjern F, Pekkari K, Gustafsson UO. The impact of perioperative fluid therapy on short-term outcomes and 5-year survival among patients undergoing colorectal cancer surgery - A prospective cohort study within an ERAS protocol. Eur J Surg Oncol 2017; 43: 1433-9.

21 Aga Z, Machina M, McCluskey SA. Greater intravenous fluid volumes are associated with prolonged recovery after colorectal surgery: a retrospective cohort study. $\mathrm{Br} J$ Anaesth 2016; 116: 804-10.

22 Brandstrup B, Tonnesen H, Beier-Holgersen R et al. Effects of intravenous fluid restriction on postoperative complications: comparison of two perioperative fluid regimens: a randomized assessor-blinded multicenter trial. Ann Surg 2003; 238: 641-8.

23 Brandstrup B, Svendsen PE, Rasmussen $M$ et al. Which goal for fluid therapy during colorectal surgery is followed by the best outcome: near-maximal stroke volume or zero fluid balance? Br J Anaesth 2012; 109: 191-9.

24 Myles PS, Bellomo R, Corcoran T et al. Restrictive versus liberal fluid therapy for major abdominal surgery. $N$ Engl J Med 2018; 7: e015358.

25 Thiele RH, Raghunathan K, Brudney CS et al. American Society for Enhanced Recovery (ASER) and Perioperative Quality Initiative (POQI) joint consensus statement on perioperative fluid management within an enhanced recovery pathway for colorectal surgery. Perioper Med (Lond) 2016; 5: 24.

26 Makaryus R, Miller TE, Gan TJ. Current concepts of fluid management in enhanced recovery pathways. Br J Anaesth 2018; 120: 376-83.

27 Srinivasa S, Taylor MH, Singh PP, Yu TC, Soop M, Hill AG. Randomized clinical trial of goal-directed fluid therapy within an enhanced recovery protocol for elective colectomy. Br J Surg 2013; 100: 66-74.

28 Srinivasa S, Kahokehr A, Soop M, Taylor M, Hill AG. Goal-directed fluid therapy- a survey of anaesthetists in the UK, USA, Australia and New Zealand. BMC Anesthesiol. 2013; 13: 5 .

29 Puckett JR, Pickering JW, Palmer SC et al. Low versus standard urine output targets in patients undergoing major abdominal surgery: a randomized noninferiority trial. Ann Surg 2017; 265: 874-81.

30 Currie AC, Malietzis G, Jenkins JT et al. Network metaanalysis of protocol-driven care and laparoscopic surgery for colorectal cancer. Br J Surg 2016; 103: 1783-94.

31 Strunden MS, Heckel K, Goetz AE, Reuter DA. Perioperative fluid and volume management: physiological basis, tools and strategies. Ann Intensive Care 2011; 1: 2. 
32 Smith PR, Baig MA, Brito V, Bader F, Bergman MI, Alfonso A. Postoperative pulmonary complications after laparotomy. Respiration 2010; 80: 269-74.

33 Arozullah AM, Daley J, Henderson WG, Khuri SF. Multifactorial risk index for predicting postoperative respiratory failure in men after major noncardiac surgery. The National Veterans Administration Surgical Quality Improvement Program. Ann Surg 2000; 232: 242-53.

\section{Supporting Information}

Additional Supporting Information may be found in the online version of this article:

Appendix S1. Receiver operating characteristic curves to define thresholds. 